\title{
sur la gestion et les finances publiques En Novembre 2020
}

\section{BUDGET DE L'ÉTAT ET DES OPÉRATEURS}

\section{$\lambda$ Politique budgétaire}

La quatrième loi de finances rectificative pour 2020 ( LFR 4)

La quatrième loi de finances rectificative de I'année, $n^{\circ}$ 2020-1473 du 30 novembre 2020 novembre, a été pubiée au JORF du 1er décembre, texte $n^{0} 1$. Elle se base sur une croissance économique à la baisse $(-11 \%$ du PIB contre $-10 \%$ dans le PLF 2021). Le déficit public est revu à $-11,3 \%$ du PIB. La dette publique atteindrait $119,8 \%$. Les annonces consécutives à la mise en place du couvre-feu puis du reconfinement et aux nouvelles mesures d'urgence conduisent à modifier la prévision du solde public de $20 \mathrm{Md} €$. La « mission » spécifique liée au dispositif d'urgence est réabondée à hauteur de 17,3 Md€. 10,9 Md€ sont ajoutés au fonds de solidarité pour les PME et les indépendants (porté de 8,5 Md€ à 19,4 Md€) ; 3,2 Md€ sont consacrés à la prise en charge du chômage partiel (dont l'enveloppe prévisionnelle est portée de 30,8 Md€ à $34 \mathrm{MdE})$; $3 \mathrm{Md}$ pour la compensation des exonérations de SS accordées à certains secteurs; 1,1 Md€ pour la prime accordée aux bénéficiaires du RSA et des APL ( $v$. Repères d'octobre) ; 2,4 Md€ pour les dépenses supplémentaires de l'Assurance maladie (matériels de santé et avancement au $1^{\text {er }}$ décembre de certaines des mesures du "Ségur de la santé »). A ce stade, le coût de la crise sanitaire est estimé à $86 \mathrm{Md} €$ d'aides et $100 \mathrm{Md}$ de pertes de recettes.

La Cour propose de réformer la gouvernance des finances publiques

La Cour des comptes a consacré un rapport publié le 18 novembre qui vise à améliorer le cadre organique de la gouvernance des finances publiques. Elle fait seize propositions d'amélioration divisées en trois grandes thématiques. La première porte sur le renforcement de la programmation pluriannuelle des finances publiques. Le deuxième volet concerne le pilotage et le rétablissement d'une vision globale des finances publiques. La Cour relève en effet que « la fragmentation du cadre budgétaire de la France traduit la complexité du cadre institutionnel, avec plus de 90000 entités relevant des trois secteurs d'administrations publiques (...) (et) (...) conduit à la mise en place d'instruments de gouvernance des finances publiques des administrations publiques à la fois complexes et lacunaires ». Le troisième objectif consiste à « assurer l'unité, I'universalité et l'efficience du budget de l'État ». À ce titre, la Cour propose de "mettre fin aux comptes spéciaux et budgets annexes pour lesquels il n'est pas démontré que la nature de leurs dépenses nécessite de déroger aux règles budgétaires ». La juridiction financière recommande également une modification de la LOLF qui permettrait une évaluation de l'exécution du budget sur trois ans.

L'avis empreint de sévérité du HCFP sur un amendement au PLF 2021

Le Haut conseil des finances publiques (HCFP) a rendu le 23 novembre un avis (2020-7) peu médiatisé mais assez singulier. Le Haut conseil avait été saisi en effet le 17 novembre sur le fondement de l'article 16 de la loi organique du 17 décembre 2012, d'une demande d'avis sur une nouvelle prévision de croissance du PIB pour 2021. Or le HCFP a estimé que cette demande était dépourvue d'éléments nécessaires lui permettant d'apprécier les prévisions macroéconomiques notamment celles déterminant les recettes publiques. Par conséquent, 
«(...) il n'est ... pas en mesure de porter une appréciation sur les hypothèses sous-jacentes à l'amendement à l'article liminaire du PLF 2021 que le gouvernement devra soumettre au vote du Parlement avant son adoption définitive ». Conscient de la difficulté d'établir des prévisions en pleine crise sanitaire, le Haut conseil note que «la prévision d'un recul de $11 \%$ du PIB en 2020 apparaît désormais prudente ».

\section{$\pi$ Dette publique}

Création d'une Commission sur l'avenir des finances publiques

Parallèlement à la présentation du PLFR 4, le ministre chargé des comptes publics, Olivier Dussopt, a annoncé le 4 novembre la création d'un groupe de travail pour le remboursement de la dette publique suite aux nombreuses dépenses occasionnées par la covid.

NDLR : Ce groupe de travail est finalement constitué et installé en décembre sous la forme d'une Commission chargée de proposer des solutions sur la manière dont la France peut redresser ses comptes publics après la crise de la Covid. Voir Repères de décembre.

\section{FinANCES LOCALES}

\section{$\pi$ Données générales sur les finances locales}

Vote de la loi de finances rectificative $n^{\circ} 4$ pour 2020

Cette loi de finances rectificative $n^{\circ} 4$, publiée le 30 novembre 2020, provisionne notamment plus de $20 \mathrm{Md}$ @ pour pallier le nouveau confinement. Mais du point de vue des collectivités elle apporte quelques nouveautés. Ainsi, sont prévues des avances remboursables à lle-deFrance mobilités $(1,175 \mathrm{Md} €$ ) et aux Autorités organisatrices de la mobilité de province (750 M€) pour compenser les pertes fiscales et tarifaires des transports publics. En outre, est également adoptée une aide exceptionnelle de 20 M€ pour le département des Alpes-Maritimes pour faire face aux conséquences sanitaires de la catastrophe naturelle liées au passage de la tempête Alex. Notons également que le Sénat avait proposé de créer une dotation d'un Md€ aux communes et à leurs groupements, pour compenser les dépenses supplémentaires liées à la crise sanitaire en 2020 mais que cette mesure n'a pas été retenue.

\section{$\pi$ Dépense publique locale}

Finances des départements et subventions d'investissement

La publication de la traditionnelle étude de la Banque postale et de l'Association des départements de France permet de rappeler quelques constats sur la situation en 2019 et l'impact de la crise actuelle en 2020 qui se traduit par l'effet de ciseaux entre une augmentation des dépenses de fonctionnement et notamment celles d'action sociale, et la baisse des recettes de fonctionnement, en repli sous l'effet de la baisse des droits de mutation à titre onéreux (DMTO). Les investissements départementaux ont continué de progresser en 2019 même si leur niveau demeure inférieur à ce qui était observé il y a une dizaine d'années. Les collectivités de plus d'un million d'habitants ont amplement participé à ce dynamisme. Les investissements devraient également augmenter en 2020, plus sous l'impulsion des subventions d'équipement versées que des dépenses d'équipement comme on aurait pu l'attendre en fin de mandat. II ressort de l'analyse approfondie des subventions d'investissement sur la période 2015/2019 qu'elles représentent un peu plus d'un tiers des dépenses d'investissement hors dette; que le bloc communal est le principal bénéficiaire de ces subventions; que les départements ruraux de moins de 250000 habitants sont ceux qui versent au bloc communal les subventions les plus importantes rapportées au nombre d'habitants.

\section{$\pi$ Transferts de l'État}

Les transferts financiers de l'État aux collectivités territoriales

Ce document édité tous les ans permet de mieux comprendre la position de l'État et les différents chiffres en matière locale. Ainsi, ce jaune financier révèle le satisfecit que l'État s'attribue par rapport aux effets de la contractualisation sur la modération de l'évolution des dépenses locales de fonctionnement. Sur la crise actuelle, l'État rappelle les montants alloués aux collectivités territoriales (5,2 Md€) dans le cadre de la troisième loi de finances rectificative de 2020. Sur la réforme des impôts de production, ce jaune qualifie les nouveaux transferts de «dynamiques ».

Meilleure prise en charge des pertes des départements

Olivier Dussopt a annoncé devant les sénateurs le 5 novembre dernier une meilleure prise en charge des pertes départementales. Ainsi, un fonds de 200 M€ pour une cinquantaine de dé- 
partements au lieu d'un chiffre initial de $115 \mathrm{M€}$ et $50 \mathrm{M} €$ d'aide en direction des petites communes qui subissent des pertes de droits de mutation à titre onéreux seront mis en place.

Mise en place des contrats de relance et de transition écologique (CRTE)

Le Gouvernement a décidé de territorialiser le plan de relance en effectuant deux démarches parallèles : d'une part inscrire le plan de relance dans le cadre de la transition écologique, et, d'autre part, simplifier la contractualisation État-collectivités en la regroupant dans ce nouveau contrat. Une circulaire du Premier ministre du 20 novembre indique ainsi que tous les territoires devront être couverts par ces nouveaux contrats d'ici le 30 juin 2021. Juridiquement, il s'agit d'une déclinaison des contrats de cohésion territoriale prévue par la loi $n^{\circ} 2019-753$ du 22 juillet 2019. En termes de périmètre, ces contrats devront être signés soit par des EPCl ou des structures plus importantes mais ne pourront excéder le cadre départemental. Ces contrats pourront inclure des fonds du plan de relance mais également la dotation de soutien à l'investissement local (DSIL) ou la dotation d'équipement des territoires ruraux (DETR].

\section{FINANCES SOCIALES}

\section{$\lambda$ Politique sociale et situation des comptes sociaux

\author{
Présentation du 4e PLFR pour 2020 \\ v. supra budget de l'État
}

Aggravation du déficit de la Sécurité sociale pour 2020 et pour 2021

Lors de la discussion du PLFSS pour 2021 au Sénat, le 10 novembre, le gouvernement a déposé un amendement réévaluant le déficit de la SS pour 2020 (régime général +FSV) à -49 Md€, contre -44,4 Md€ prévus dans le projet initial ( $v$. Repères de septembre). Le déficit de la branche Maladie serait désormais de -33,7 Md€, compte tenu des dépenses supplémentaires induites par l'avancement de la deuxième tranche des mesures salariales prévues par le «Ségur de la santé » (déjà pris en compte en première lecture à l'Assemblée nationale) et par les conséquences de la deuxième vague de la pandémie ( achat de tests PCR, surcroît de dépenses dans les EHPAD...). Les autres branches verraient leur solde se dégrader par rapport à la prévision initiale, du fait des pertes de recettes induites par le reconfinement, en particulier la branche Vieillesse dont le déficit 2020 passerait de -7,9 à-8,6Md€. Parallèlement le déficit prévisionnel pour 2021 a été relevé à -35,7 Md€.

Des mesures en faveur des entreprises, des précaires et des jeunes

En présentant le calendrier du déconfinement le 24 novembre, le Président de la République annoncé de nouveaux efforts en faveur des entreprises ainsi que des travailleurs précaires et des jeunes. Le dispositif en a été détaillé par le Premier Ministre le lendemain. Les entreprises qui resteront fermées par mesure administrative au moins jusqu'au 20 janvier 2021 ( bars, restaurants, salles de sport, discothèques, activités des stations de sport d'hiver...), pourront opter, au titre de l'aide du Fonds de solidarité pour les PME et les indépendants, entre soit l'allocation forfaitaire mensuelle de $10000 €$, soit une aide représentant $20 \%$ du chiffre d'affaires du même mois de l'année précédente, dans un plafond de $100000 €$; le bénéfice de cette aide sera étendu aux entreprises restées ouvertes (hôtellerie, événementiel..) mais ayant subi une chute de chiffre d'affaires de $50 \%$ par rapport à la normale, ainsi qu'aux fournisseurs de ces entreprises. Le coût de ces mesures (de l'ordre de 3,5 Md€) sera pris en charge dans le PLFR 4 (cf supra). Mais l'annonce la plus commentée concerne les travailleurs précaires sur contrats courts (extras, saisonniers, intérimaires, notamment de l'hôtellerie-restauration) qui n'auraient pas pu « recharger leurs droits » en 2020, ils bénéficieront d'une garantie de ressources de $900 €$ par mois nets des autres allocations (RSA ....) ; le coût de la mesure est estimé à 1,5 Md€ pour 350000 bénéficiaires. S'agissant des jeunes, outre une mise en œuvre volontariste du plan «Un jeune, une solution » (v.Repères de juillet), tout jeune pourra bénéficier d'une allocation de $500 €$ par mois en fonction de sa situation et le nombre de places dans le dispositif d'accompagnement de la " garantie de ressources », déjà augmenté en juillet, sera porté à 200000 . Enfin, 20000 jobs de tutorat pour pallier le décrochage en université seront créés pour 4 mois, de même que le budget des CROUS sera augmenté.

\section{$\pi$ Retraites}

\section{Réformer les retraites en temps de crise}

Une note de l'Institut Montaigne du $1^{\text {er no- }}$ vembre analyse l'impact de la crise sanitaire sur la situation des régimes de retraite. Partant du dernier point d'étape du COR (v. Repères d'octobre) et de la situation des finances publiques telle qu'elle ressort du PLF 2021, I'Institut table 
sur un déficit de ceux-ci de l'ordre de -30 Md€ en 2030. Reprenant les constats largement partagés lors du débat sur le projet gouvernemental de l'hiver dernier sur le caractère généreux des régimes français au regard de la richesse nationale et relevant que la situation des retraités a été peu affectée par la crise sanitaire, la note estime que la réforme systémique envisagée est aujourd'hui de second rang par rapport à la question de la soutenabilité financière. Présentant les solutions traditionnellement envisagées pour jouer sur les grands paramètres (niveau des pensions, niveau des cotisations, âge de départ ou durée de cotisation), la note préconise notamment de recycler les économies qui seront réalisées sur les régimes spéciaux pour améliorer les droits des chômeurs à venir et de laisser chacun choisir individuellement entre l'allongement de sa durée de cotisation ou la réduction de sa pension. À la fin de novembre, le gouvernement paraissait divisé sur l'opportunité de reprendre la réforme d'ici la fin du septennat.

Le rapport définitif du Conseil d'orientation des retraites

Le Conseil d'orientation des retraites a rendu le 21 novembre le rapport définitif sur l'évolution des régimes de retraites à l'horizon 2030 que lui avait commandé le Premier Ministre et pour lequel une note d'étape avait été présentée en octobre. A partir de quatre scénarios d'évolution du PIB (entre $1 \%$ et 1,8 \% ), I'évolution tendancielle fait apparaitre que les dépenses du système de retraite resteraient stabilisées autour de 13,8 \% du PIB en 2030 , que I'âge moyen effectif du départ en retraite passerait à 63,3 ans (contre 62,1 ans en 2018), et que le niveau de vie moyen des retraités resterait à peu près à parité avec le niveau de vie moyen de la population. Selon les conventions habituelles sur le financement des régimes spéciaux et en croisant avec les scénarios de croissance, le besoin de financement du système de retraite en 2030 irait de $-0,3 \%$ à $-1 \%$ du PIB (soit un déficit compris entre $-8 \mathrm{Md}$ et $-27 \mathrm{Md}$ ). Enfin, dans la perspective d'un retour à l'équilibre du système dès 2025, le COR fait plusieurs scénarios en jouant sur les paramètres traditionnels d'équilibrage des régimes (I'âge de départ obligatoire combiné avec la durée d'assurance, le niveau des pensions et le taux de cotisation). Ces prévisions auraient donc plutôt tendance à relativiser le problème du financement des retraites à moyen terme, au regard d'une situation de départ très dégradée, puisque les prévisions pour 2020 seraient d'un déficit de 23,4 Md€ (1,1 du PIB) susceptible d'être alourdi de 1,6 Md€ du fait du deuxième confinement.

\section{\ Solidarité, Lutte contre la pauvreté/ Revenu universel}

Hausse des bénéficiaires des minimas sociaux et de la prime d'activité

L'enquête annuelle de la DREES, publiée le 10 novembre, fait apparaître une légère augmentation en 2019 du nombre d'allocataires des dix minima sociaux $(+1,2 \%)$, concentrée surtout sur les effectifs du minimum vieillesse suite à l'augmentation importante du plafond de ressources de celui-ci. Le nombre de bénéficiaires du RSA a faiblement augmenté en 2019 (+ 0,6\%). En revanche, le nombre d'allocataires de la prime d'activité a explosé en 2019 (+ 42,7\%) du fait des mesures de revalorisation intervenues fin 2018 suite à la crise des «Gilets jaunes », le montant de la dépense s'élevant à 9,8 Md€ (contre 5,6 Md€ en 2018).

Explosion des dépenses du RSA en 2020, I'État pourrait reprendre la main

Selon des informations publiées par les Échos, le 10 novembre, les dépenses des départements liées au RSA pourraient augmenter de 1 Md€́ en 2020, mettant certaines collectivités en grande difficulté. Le Premier Ministre a écrit au président du Conseil départemental de la Seine-Saint-Denis que l'État pourrait, à titre expérimental, prendre intégralement en charge le RSA de ce département en 2022, conduisant ainsi à la renationalisation de ce qui est considéré par beaucoup d'analystes comme une dépense de solidarité nationale. Les dernières statistiques de novembre 2020 de la CNAF confirment cette évolution, le nombre de foyers allocataires pouvant dépasser 2 millions à l'automne.

Quels effets de la pandémie sur le niveau des inégalités en France?

Une étude de l'Institut des politiques publiques et du Cepremap du 17 novembre analyse l'impact de la crise sanitaire sur le niveau de vie des ménages et l'effet des mesures prises par le gouvernement lors du premier confinement. Si, selon les données retranscrites par les Échos, globalement, le pouvoir d'achat des Français paraît avoir été peu affecté $(-0,5 \%$ selon le gouvernement en septembre), les ménages les plus modestes ont été plus impactés (moindre possibilité de recours au télétravail, prépondérance des contrats courts). Mais cette situation a pu être corrigée par les mesures gouvernementales; pour les revenus les plus modestes: 
revalorisation de l'allocation de rentrée scolaire et aides exceptionnelles aux bénéficiaires d'aides sociales; pour les indépendants et les PME : dispositif du Fonds de solidarité (fortement revalorisé en octobre). Le dispositif de chômage partiel a surtout bénéficié aux classes moyennes, pour lesquels la perte de revenu a été évaluée à - $0,4 \%$ entre mars et juillet. Toutefois ce constat va nécessairement évoluer du fait de la prolongation de la crise et du risque de l'accroissement de la précarité des jeunes. Par ailleurs, I'Observatoire des inégalités vient de présenter son rapport 2020,

\section{$\pi$ Assurance chômage}

Le Conseil d'État met à mal la réforme de I'Assurance chômage

Même si le Gouvernement avait annoncé le report de la réforme de l'assurance chômage au $1^{\text {er }}$ avril 2021 (v. Repères d'octobre), des possibilités d'assouplissement et une prolongation du versement de l'allocation pour les chômeurs arrivant en fin de droits, il entendait toujours mettre en œuvre les dispositions restantes de sa réforme. Mais le Conseil d'État, le 25 novembre, a compliqué cette perspective en annulant, sur recours d'organisations de syndicats et d'employeurs, deux volets essentiels du décret du 26 juillet 2019 : le mode de calcul du salaire journalier de référence (au motif d'atteinte au principe d'égalité puisque pour un même nombre d'heures travaillées le salaire de référence pourrait varier du simple au quadruple) et la quasi intégralité du bonus/malus des cotisations (pour un motif de procédure). II reste que $d^{\prime}$ autres dispositions comme la nouvelle durée d'affiliation et la dégressivité des allocations pour les cadres ont été validées.

\section{FisCALITÉ ET PROCÉDURES FISCALES}

\section{$\pi$ Politique fiscale}

LFR 4 : des recettes fiscales rehaussées, sans dispositions fiscales nouvelles

Comme cela a été souligné dans Les Échos lors de la présentation du 4 PLFR par le Gouvernement : «les rentrées fiscales résistent malgré le reconfinement ». Le rebond de l'économie française de l'été a amélioré les rentrées fiscales, atténuant quelque peu le «choc » budgétaire pour l'exercice 2020. Les recettes fiscales nettes sont prévues dans la 4e LFR (L. n²020-1473, 30 nov. 2020) à un niveau de 249,3 Mds€, en hausse de 2,5 Mds€ environ par rapport au niveau révisé lors de la présentation du PLF pour 2021, mais toujours en diminution importante de 43,7 Mds€ (soit $15 \%$ ) par rapport au niveau prévu en LFI 2020 et, pour la première fois depuis la deuxième guerre mondiale, inférieur au montant net des émissions de dettes à moyen et long terme. Comme le note le rapporteur général au Sénat, « alors qu'un couvrefeu dans certaines zones, puis un confinement sur l'ensemble du territoire ont été annoncés par la suite ». Les trois recettes fiscales les plus impactées par la crise liée à la Covid-19, en rapport des prévisions de la LFI 2020, sont I'IS (-15,5 Mds€), la TVA (-14 Mds€) et la TICPE $(-6,1 \mathrm{Mds} €)$. En effet, on observe que le produit de I'IR (-2,3 Mds€) est moins fortement dépendant des évolutions de la conjoncture économique. Conformément à une nouvelle pratique, initiée en 2019 par le Gouvernement, la 4e LFR pour 2020 ne contient aucune disposition fiscale dans un souci de respect des autorisations parlementaires formulées en LFI mais aussi de stabilité de la législation fiscale. Le Conseil constitutionnel n'a pas été saisi de sa constitutionnalité.

\section{$\pi$ TVA}

La TVA du futur et à l'ère du digital en France

Un rapport de la DGFiP, intitulé « La TVA à l'ère du digital en France », a été remis au Parlement, après une consultation des principaux assujettis concernés par la réforme. II présente différentes pistes de son déploiement. Le Gouvernement a proposé la généralisation de la facturation électronique à toutes les transactions entre entreprises entre le $1^{\text {er }}$ janvier 2023 et le $1^{\text {er jan- }}$ vier 2025 dans le PLF 2021. Le principal objectif de cette réforme est le renforcement de la lutte contre la fraude fiscale et la diminution de l'écart de TVA au moyen de recoupements automatisés. L'obligation de facturation électronique entre entreprises est accompagnée d'une obligation complémentaire de transmission de données.

\section{$\pi$ Impôt sur les sociétés}

\section{La taxe « GAFA » sera prélevée en 2020} par la DGFiP

Le Gouvernement avait décidé de suspendre les demandes d'acompte de la taxe "GAFA » en attendant l'issue des négociations sur la fiscalité des multinationales dans le cadre de I'OCDE (cf. Repères, janvier 2020). Comme celles-ci n'ont pas abouti en raison de l'oppo- 
sition de l'administration Trump, la DGFIP a confirmé en novembre que les entreprises assujetties à la taxe "GAFA » avaient reçu un avis d'imposition pour le versement des acomptes de 2020.

Un litige fiscal onéreux avec Orange SA pour les finances de l'État?

La société française de télécommunications Orange SA conteste un redressement fiscal qui lui a été infligé après sa réorganisation en 2005. Si le Conseil d'État lui donne gain de cause, ce qui n'est pas à exclure au regard des conclusions du rapporteur public, le coût dépasserait 2,6 Mds€ pour les finances de l'État. A la suite de la fusion-absorption de sa holding Cogecom dans laquelle ses acquisitions étaient logées, l'opérateur français de télécommunications, encore dénommé France Télécoms, a déduit de son bénéfice 11,5 Mds€, correspondant aux reprises de provisions passées au cours des années précédentes. L'administration fiscale, qui contestait cette déduction liée à des dépréciations d'actifs, a réclamé qu'Orange SA lui verse 1,9 Mds€ ainsi que des intérêts de retard, ce qui a été contesté devant la CAA de Versailles puis devant le Conseil d'État par ladite société. Affaire à suivre...

\section{$\lambda$ Impôt sur le capital}

Taxe sur les immeubles de $3 \%$ : les précisions de la Cour de Cassation

La Cour de Cassation (Cass. Com., 4 nov. 2020, Société Lupa SA, pourvoi n¹8-11.771) vient de censurer la Cour d'Appel de Paris qui avait jugé s'agissant de la taxe de $3 \%$ sur les immeubles que les seules sanctions applicables pour dépôt tardif de la déclaration sont celles prévues aux articles 1727 et 1728 du CGI, soit un intérêt de retard et une majoration du montant des droits mis à la charge du contribuable et non le paiement de la taxe elle-même.

Inconstitutionnalité de la taxe de cessions d'objets précieux hors UE

Selon le Conseil constitutionnel, I'exclusion du champ de la taxe forfaitaire des cessions à titre onéreux d'objet précieux réalisées en dehors de I'UE (paragraphe II de l'art. $150 \mathrm{VI}$ du CGI issu des dispositions de la LFI 2014) est contraire à la Constitution (Cons. const., décis. n²020-868 OPC, 27 nov. 2020, M. Louis-Christophe L.), et notamment au principe d'égalité devant la loi.

\section{Contrôle fiscal et contentieux fiscaux}

Le coût des contentieux fiscaux en nette augmentation pour l'État

En 2020, le coût budgétaire associé aux contentieux fiscaux de série, c'est-à-dire ceux pour lesquels plusieurs contribuables ont déposé des recours, et donc les plus coûteux pour les finances de l'État, pourrait connaître une forte augmentation, et atteindre 3,9 Mds€. La prévision initiale s'établissait à 2,6 Mds€. La réévaluation s'explique pour l'essentiel par celle des dégrèvements prévus dans le cadre du contentieux des organismes de placement collectif en valeurs mobilières (OPCVM) à hauteur de 0,9 Mds€. Dans une moindre mesure, cette réévaluation s'explique également par la revalorisation en cours d'année du coût du contentieux «Précompte » à hauteur de 0,3 Mds€ pour 2020. En 2021, le coût budgétaire associé aux contentieux fiscaux devrait reculer, mais se maintenir à des niveaux élevés, du fait de la poursuite de certains de ces contentieux de série (2,5 Mds€).

\section{Management public}

\section{$\pi$ Politique de modernisation}

Une circulaire du 18 novembre sur le suivi des priorités du Gouvernement

Le Premier ministre a adressé aux membres du Gouvernement une circulaire $n^{\circ}$ 6230/SG datée du 18 novembre 2020 sur le suivi de l'exécution des priorités gouvernementales. Le pilotage interministériel est renforcé : désignation d'un membre du gouvernement comme chef de file responsable, chefs de projet, suivi par la ministre de la transformation et de la fonction publiques, par les cabinets et par la direction interministérielle de la transformation publique, point régulier en partie $D$ du conseil des ministres intitulée "résultats». Un pilotage territorial est confié aux préfets qui associeront les parties prenantes, dont les parlementaires. Un suivi des réformes prioritaires sera assuré au moyen d'un tableau de bord unique et territorialisé tenu par la direction interministérielle de la transformation publique et au moyen d'un baromètre des trente politiques prioritaires du Gouvernement qui sera présenté régulièrement en conseil des ministres par la ministre de la transformation et de la fonction publiques.

\section{Les trois priorités de la Ministre}

Dans une communication au conseil des ministres du 10 novembre, Amélie de Montchalin, ministre de la transformation et de la fonction 
publiques, a présenté trois orientations prioritaires. Premier axe : Améliorer les recrutements et favoriser l'égalité des chances dans la fonction publique (amélioration du taux de réussite dans les classes préparatoires, développement de l'apprentissage, respect des obligations au titre du recrutement de travailleurs handicapés, égalité entre les femmes et les hommes avec un objectif de $40 \%$ des premières nominations aux emplois d'encadrement supérieur). Deuxième axe: Améliorer le service rendu aux citoyens dans le cadre d'un programme "service plus » (porter le taux de satisfaction des usagers à $77 \%$ en 2022, simplifier, numériser $100 \%$ des démarches les plus fréquentes). Troisième axe : Déployer France Connect qui permet d'accéder à une multitude de services publics par un identifiant unique (objectif 2022 : 30 millions d'utilisateurs et 1300 services accessibles). Ces priorités sont assorties d'indicateurs et feront l'objet d'un suivi interministériel dans le cadre d'un « baromètre des résultats de l'action publique».

\section{$\pi$ Administration numérique}

\section{Accélération du déploiement de France Connect}

Le gouvernement a décidé d'accélérer le déploiement du service France Connect, ouvert en 2016 et développé par la Dinum, qui permet d'accéder à une multitude de services par un identifiant unique et de simplifier les démarches des utilisateurs par l'échange automatique de données entre ces services. II compte actuellement 19 millions d'utilisateurs et 750 services accessibles. Une campagne de promotion a été lancée pour améliorer sa notoriété. Fin 2021, avec la nouvelle identité numérique sécurisée préparée par le ministère de l'intérieur, il pourra être utilisé par de nouveaux services, notamment dans les secteurs de la santé et de la justice. L'objectif 2022 est que l'ensemble des sites internet publics offrent le bouton France Connect et qu'il soit utilisé par 30 millions d'usagers de 1300 services.

\section{Succès de Tousanticovid}

A la fin du mois de novembre, le dispositif de signalement des cas positif était téléchargé par 9,8 millions d'utilisateurs et avait assuré 13866 notifications à des cas contact.

\section{Des conseillers numériques France ser-} vices

Le secrétariat d'État chargé du numérique a annoncé le 17 novembre la création d'un réseau de 4000 conseillers numériques auprès des col- lectivités territoriales et des associations pour organiser des ateliers d'initiation et de perfectionnement au numérique sur tout le territoire. Ce dispositif renforce les outils développés dans le cadre de la stratégie nationale pour un numérique inclusif.

\section{$\lambda$ Ministère des finances}

\section{Le rapport d'activité 2019 du SG du MEFIR}

Le rapport d'activité du secrétariat général du ministère de l'économie, des finances et de la relance pour 2019 consacre une partie importante à... 2020 : la gestion de la crise sanitaire a mis le SG à rude épreuve pour protéger, équiper, informer... Le SG, qui regroupe 2500 agents, affiche quatre missions permanentes: conduire la transformation du ministère, animer le dialogue social, gérer les fonctions supports, assurer le bon fonctionnement des services centraux. Parmi les actions présentées : le plan Bercy vert, les nouveaux usages du numérique, la contribution du Bercy lab à une soixantaine de projets, les nouvelles stratégies immobilières, la mise en place des contrats objectifs/moyens.

\section{$\pi$ Fonction publique}

\section{Le rapport sur la fonction publique}

Le rapport annuel sur la fonction publique apporte son lot d'informations. Sur les politiques et pratiques de ressources humaines, un titre fait le point sur la mise en œuvre de la loi du 6 août 2019 de transformation de l'action publique, sur la crise sanitaire et la santé au travail, sur l'égalité de traitement et sur les importantes évolutions en matière de ressources humaines. Les «faits et chiffres » nous apprennent qu'en 2018, la hausse de l'emploi public est portée par la conversion des contrats aidés en contrats de droit public, qu'elle est moins forte dans la FP hospitalière que dans les autres secteurs, que le nombre de départs à la retraite baisse pour la première fois depuis 2015, que le salaire net moyen mensuel atteint $2.995 €$. Un dossier sur l'attractivité dans la fonction publique et de nombreuses fiches thématiques complètent ce rapport.

Les nouveaux organismes paritaires de la fonction publique de l'État

Le décret $n^{\circ} 2020-1427$ du 20 novembre 2020, pris en application de l'article 4 de la loi $n^{\circ}$ 2019-828 du 6 août 2019 de transformation de la fonction publique, organise les nouveaux comités sociaux d'administration (CSA) qui, à 
I'issue des élections professionnelles de 2022, résulteront de la fusion des comités techniques paritaires et des comités d'hygiène, de sécurité et des conditions de travail (CHSCT). Le CSA est obligatoirement consulté sur les projets de texte réglementaire relatifs au fonctionnement et à l'organisation des services, les projets de lignes directrices de gestion relatives à la stratégie pluriannuelle de pilotage des ressources humaines, aux orientations générales en matière de mobilité et aux orientations générales en matière de promotion et de valorisation des parcours professionnels (article 48). Une formation spécialisée en matière de santé, de sécurité et de conditions de travail sera obligatoirement créée lorsque l'effectif dépassera 200 agents. Le décret définit la composition, l'organisation et les règles de fonctionnement de ces instances. Le décret $n^{\circ} 2020-1426$ du 20 novembre 2020 relatif aux commissions administratives paritaires dans la fonction publique de I'État, modifiant le décret $n^{\circ} 82-451$ du 28 mai 1982, réorganise les CAP qui, en vertu de la loi de transformation de la fonction publique, ne sont plus compétentes en matière de mutation

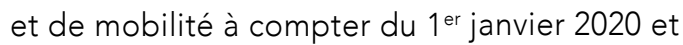
en matière $d$ 'avancement et de promotion dès le $1^{\text {er }}$ janvier 2021. Il instaure les règles de création des CAP par catégorie hiérarchique et non plus par corps.

\section{EUROPE}

\section{Politique européenne}

\section{Aides d'État : la Commission toujours aussi conciliante}

Tout au long du mois de novembre, la Commission européenne a accepté plusieurs mesures nationales motivées par la lutte contre les effets de la pandémie de Covid-19 : I'indemnisation du secteur des transports aux Pays-Bas, le soutien roumain aux investissements pour le chauffage urbain fondé sur des sources d'énergie renouvelables, un mécanisme d'appel d'offre pour la fermeture anticipée des centrales à charbon et une aide couvrant les frais fixes des entreprises touchées par la covid-19 en Allemagne, mais aussi au Luxembourg et en Autriche concernée aussi par une aide au transporteur ferroviaire, ou encore une aide en capital aux grandes entreprises danoises.

\section{Pacte de stabilité}

Semestre européen - paquet d'automne : la Commission se focalise sur la reprise
Le 18 novembre, la Commission européenne a présenté son paquet d'automne pour la politique économique, comprenant ses avis sur les projets de plan budgétaire (PPB) des États membres de la zone euro pour 2021 et ses recommandations pour la zone euro. Comme en juillet 2020, ses recommandations sont uniquement qualitatives, compte tenu de la suspension (l'activation de la clause dérogatoire générale du pacte de stabilité et de croissance) et favorisent le soutien à l'activité économique. L'examen du cas de la Roumanie (pour laquelle une procédure pour déficit excessif - couramment appelée procédure de sanction - a été ouverte en avril 2020) a été repoussé au printemps 2021.

\section{$\lambda$ Budget européen}

La Cour des comptes européenne valide les comptes 2019 mais reste critique sur les dépenses

Le 10 novembre 2020, le président de la Cour des comptes européenne a présenté devant la Commission du contrôle budgétaire son rapport 2019. Le même jour, la Cour a validé les comptes 2019, ce que la Commission a salué. La Cour a cependant considéré que les «dépenses à haut risque» comportent trop d'erreurs, liées à des critères d'éligibilité ou autres règles complexes. II s'agit par exemple de projets de recherche ou d'investissements dans le développement régional ou rural. Le plus souvent, des bénéficiaires se révèlent inéligibles ou ne respectent pas les règles en matière de marché public. Le niveau d'erreur est estimé à 4,9\% des montants, contre $4,5 \%$ en 2018 . Neuf cas ont été signalés à l'Office européen de lutte antifraude (OLAF), qui a ouvert cinq enquêtes.

Veto magyaro-polonais au budget et au plan de relance

Le 16 novembre 2020, après avoir menacé, les premiers ministres hongrois et polonais ont bloqué l'adoption du plan de relance et du cadre financier 2021-2028 (et donc du budget européen 2021) qui avaient fait l'objet d'un accord entre le Parlement et la présidence allemande du Conseil le 10 novembre. Ils s'opposent au règlement instaurant une conditionnalité des fonds structurels au respect de l'article 2 du Traité sur l'Union européenne qui fait de l'État de droit une valeur commune des États membres. Le règlement peut être adopté à la majorité qualifiée, contrairement au cadre financier et au plan de relance qui requièrent I'unanimité. Dans une déclaration du 18 novembre, la Conférence des présidents du Par- 
lement européen a refusé toute concession sur l'État de droit et a appelé le Conseil à adopter le paquet. Réunis à nouveau le 25 novembre, les dirigeants hongrois et polonais ont proposé à leur partenaire de ne pas attendre plus longtemps pour adopter le Cadre financier et le plan de relance et, pour cela, de repousser les discussions sur le règlement conditionnant les financements européens à l'État de droit. Le 26 novembre, le philanthrope George Soros a publié une tribune dans les Échos encourageant l'Europe à leur tenir tête sur l'État de droit.

NDLR : Le Conseil puis le Parlement européen ont finalement approuvé le cadre financier pluriannuel et le plan de relance: voir Repères de décembre.

\section{$\pi$ Euro}

Un rapport de l'OFCE sur la dette publique et les banques centrales

Le 9 novembre, I'OFCE a publié un rapport des économistes Christophe Blot et Paul Hubert examinant les options possibles pour la Banque centrale européenne. La politique d'assouplissement quantitatif (QE) permet de réduire la charge d'intérêts mais une monétisation des dettes souveraines, donc une création de monnaie, serait plus efficace en termes de stabilisation de la croissance. Elle exigerait toutefois une plus forte (mais pour l'heure improbable) coordination des politiques budgétaires. Quant à l'annulation de dettes publiques, elle provoquerait une hausse des taux d'intérêt.

\section{$\pi$ Fiscalité}

Le Conseil adopte des conclusions pour une fiscalité équitable et efficace

Le 27 novembre, les États membres ont adopté des conclusions prônant une politique fiscale européenne équitable et efficace dans l'économie de plus en plus mondialisée et numérisée du XXle siècle. Elle appuie le travail de l'OCDE notamment contre l'érosion de la base d'imposition et le transfert de bénéfices (BEPS) et vise une solution multilatérale pour taxer l'économie numérique et mondialisée.

\section{INTERNATIONAL}

\section{$\pi$ Relations multilatérales}

$\mathrm{OMC}$ : report de la réunion de désignation de la nouvelle DG

Le 9 novembre, en raison de l'opposition des États-Unis à la candidature de la Nigériane Ngozi Okonjo-Iweala, la mieux positionnée, le Conseil général de l'OMC a reporté sa réunion de désignation de la nouvelle Directrice générale.

Boeing : I'UE met en place des contre-mesures à l'égard des exportations américaines

Suite à l'autorisation obtenue de I'OMC le 26 novembre, la Commission européenne a annoncé augmenter les droits de douane sur les importations européenne jusqu'à concurrence de 4 milliards d'euros.

Aurélien BAUDU (Fiscalité et procédure fiscale), Fabrice BIN (Europe, international), Jean-François CALMETTE (budget de l'État et des opérateurs),

Matthieu HOUSER (finances locales),

Michel LE CLAINCHE (comptabilité publique, management public, coordination), Yves TERRASSE (finances sociales).
Retrouvez sur www.gestionfinancespubliques. info :

- I'intégralité des REPÈRES d'actualité des finances et de la gestion publiques depuis 2015 -le 15 du mois, les REPÈRES d'actualité du mois précédent

Dans ce numéro : le " best of » de l'actualité des finances et de la gestion publique 2020. 